\title{
Reconstruction Technique Based on the Theory of Compressed Sensing Satellite Images
}

\author{
Wang Feng*, Chen Feng-wei and Wang Jia
}

School of Software, North China University of Water Resources and Electric Power, Zhengzhou 450011, China

\begin{abstract}
Owing to the characteristics such as high resolution, large capacity, and great quantity, thus far, how to efficient store and transmit satellite images is still an unsolved technical problem. Satellite image Compressed sensing (CS) theory breaks through the limitations of traditional Nyquist sampling theory, it is based on signal sparsity, randomness of measurement matrix and nonlinear optimization algorithms to complete the sampling compression and restoring reconstruction of signal. This article firstly discusses the study of satellite image compression based on compression sensing theory. It then optimizes the widely used orthogonal matching pursuit algorithm in order to make it fits for satellite image processing. Finally, a simulation experiment for the optimized algorithm is carried out to prove this approach is able to provide high compression ratio and low signal to noise ratio, and it is worthy of further study.
\end{abstract}

Keywords: Compressed Sensing, Optimization Algorithm, Random Sampling, Satellite Image Compression, Sparse representation.

\section{INTRODUCTION}

Meteorological data has been widely used in military and civil fields. In order to apply meteorological data in the climate operation, a continuous climate data sets must be formed, which requires continuously acquire massive amount of meteorological data. At present, our country mainly has two kinds of meteorological data: satellite cloud images and radar signals. At the past, those materials are mainly images, with large size and amount. Recent years, these materials have generally been digitized. Transmission speed and storage occupation have been greatly improved compared to the previous image files. However, along with the continuous development of Chinese economy and meteorological technology, size of those digitalized climate data is still remains big, so the meteorological department has being plagued by how to remote transmit and store these data.

In recent years, compression sensing theory that proposed in the field of signal processing has pointed out: when the signal has sparsity, by solving an optimized problem, accurate signal or approximate reconstruction can be achieved by signal measurement value which is collected far lower than Nyquist sampling rate $[1,2]$. Due to the CS theory can effectively reduce the cost of signal transmission, storage and process, many scholars and research institutions have carried out the research of CS theory base on graphic processing.

\section{COMPRESSION SENSING THEORY}

The Compression sensing theory points out that a measurement matrix which is unrelated to transformation matrix can be designed on the premise of signal sparse or compressible, linear project the transformation coefficient to lowdimension observation vector, and reconstruct original highdimensional signal accurately from low-dimension observation vector through solve sparse optimization problem.

Suppose there is a sparse signal $\mathrm{X}$, length is $\mathrm{N}$, measurement vector is $\Phi$, then the mathematical expression is:

$\mathrm{Y}=\Phi \mathrm{X}=\Phi \Psi \alpha=\theta \alpha$

In this expression, $\mathrm{X} \varepsilon \mathrm{R}^{\mathrm{N}}$ is original signal;

$\Phi \varepsilon R^{\mathrm{MxN}}(\mathrm{M} \ll \mathrm{N})$ is measurement matrix; $\mathrm{X}=\Psi \alpha$ is $\mathrm{X}$ 's sparse representation under certain transformation basis $\Psi \varepsilon R^{\mathrm{MxN}} ; \theta=\Phi \Psi$ is sensing matrix; ${\mathrm{Y} \varepsilon \mathrm{R}^{\mathrm{M}}}^{\mathrm{M}}$ is the measurement value obtained by $\mathrm{X}$ linear projection under measurement

Matrix $\Phi$, is the low-dimensional measurement vector of MX1 dimension.

Most of the images themselves are not sparse under the natural state, however, on the premise of signal $\mathrm{X}$ is sparse, there is:

$\mathrm{Y}=\Phi \mathrm{X}=\Phi \Psi \alpha=\theta \alpha$

In this expression, $\theta=\Phi \Psi$ is sensing matrix of MXN, as shown in Fig. (1).

The signal acquisition and process in the traditional coding mainly include four parts: sampling, compressing, transmission and decompression. As shown in Fig. (2). In the traditional decoding method, because of the frequency of signal sampling shall be not less than two times of the signal 


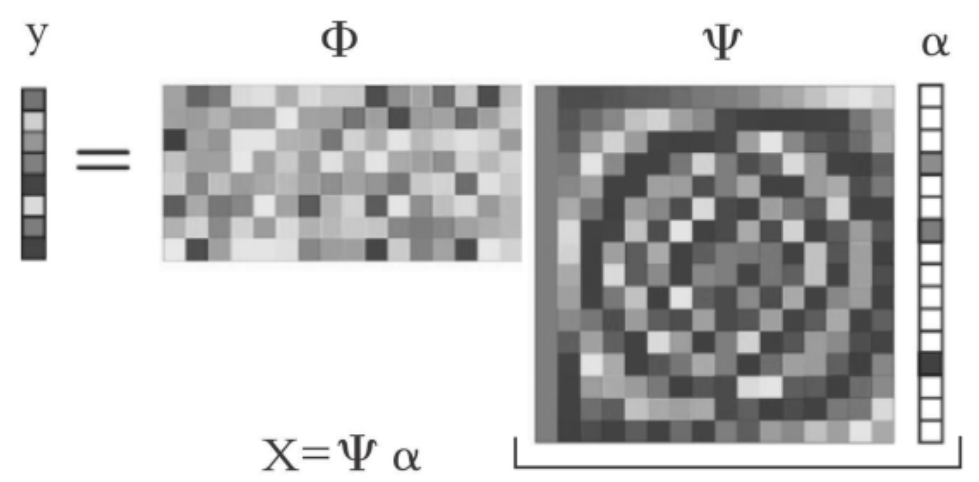

Fig. (1). Compression sensing linear measurement process.

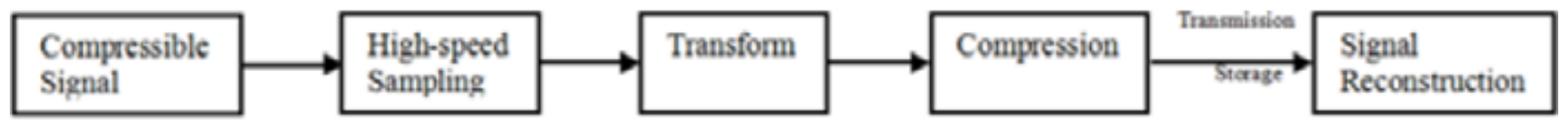

Fig. (2). Traditional signal decoding process.

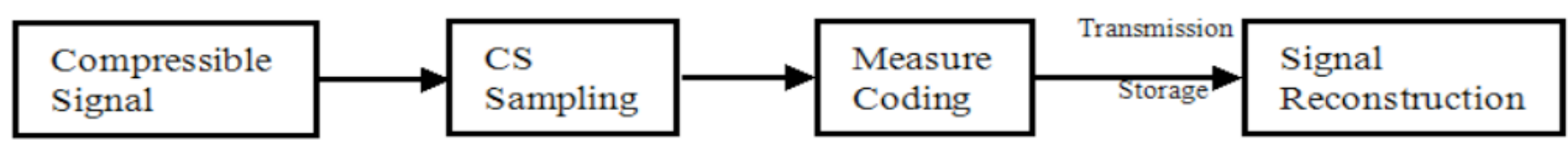

Fig. (3). CS theory signal decoding process.

bandwidth, makes the hardware equipment and network transmission face a lot of pressure, and it is also a waste of sampling resources in the compression process after such high-speed sampling.

Unlike the traditional coding method, CS theory is able to sampling and compressing signal at the same time, and takes advantage of signal sparse, coding signal nonadaptively. As shown in Fig. (3). Large-scale data set signal compression method based on compression sensing breaks through the bottleneck of traditional method of signal compression in aspects such as large amount of sampling data, uncontrolled compression accuracy, large storage space and so on, its outstanding advantage is less sampling points, fast compression speed and higher compression ratio, as well as minimal loss of information after restore the original data.

\section{SATELLITE CLOUD IMAGE PROCESS BASED ON COMPRESSION SENSING}

Due to the large data size and quantity of satellite cloud image, there are huge pressure on data transform and storage, the emerge of compression sensing theory has efficiently improved the situation, the general process of CS theory used in image processing field is: sparse original signal to obtain corresponding sparse matrix, and then set numbers which below threshold in the sparse matrix as 0 , to obtain sparse coefficient.

Design observation matrix according to the Restricted Isometry Property (RIP) criterion and obtain measured values under this matrix, finally obtain original image reconstruction by solve the optimal norm problem.

\subsection{Sparse Processing for Satellite Cloud Image}

Satellite cloud image, as important climate information, has great varieties, for instance, visible cloud image, infrared cloud image, water vapor image etc. Different types of satellites have great differences from image characteristics to image effect. Compare visible cloud image with infrared cloud image, for example, there are great difference in appearance, at the same time, similarities also exist.

In addition, from the perspective of color composition, colors of satellite cloud image are mixed and welldistributed, but the rules of composition of two-dimensional image (landscape, figure, etc.) is highlight the center,vital scene and color is generally concentrated in the center of the image.

Thus, the satellite cloud picture can not be equivalent to ordinary two-dimension image to sparse, but should find a proper sparse mode. This paper is aim to find out a suitable sparse method and reconstruction method for satellite cloud image based on predecessors' research. In recent years, Mallat [3], Taubman [4], Xiong [5] and so on, have successively carried out image compression tests by use wavelet transform method, and great results have been achieved. For any square integrable signal $\mathrm{f}(\mathrm{t})$,cloud expand in wavelet:

$$
\mathrm{f}(t)=\sum_{\mathrm{k}} \beta_{j_{0}} \phi_{j_{0}}(\mathrm{k})+\sum_{\mathrm{j}=\mathrm{j}_{0}}^{\infty} \sum_{\mathrm{k}} \alpha_{\mathrm{j}_{\mathbf{q}} \mathrm{k}} \Psi_{\mathrm{j}_{\mathbf{q}} \mathrm{k}}(\mathrm{t})
$$


In this expression, $\varphi_{\mathrm{j} 0}, \mathrm{k}(\mathrm{t})$ is scale signal, $\Psi_{\mathrm{j}, \mathrm{k}}(\mathrm{t})$ is wavelet signal, they have different expressions according to different wavelet transform. Wavelet transform obtains local compromise of time-frequency by sacrifice part of the orientation performance of frequency domain, provides accurate time domain orientation and frequency domain orientation at the same time.

For a climate image, most of the pixel values are not zero, but when the image transforms to wavelet domain, the absolute value of most of the wavelet coefficient are close to zero, and limited big coefficients are able to show most information of the original image. Therefore, this research will employ wavelet transform to carry out sparse transform for satellite cloud image.

\subsection{The Selection of Measurement Matrix}

An important part of compression sensing theory is measurement matrix. A measurement matrix which is suitable for satellite cloud picture not only can project original compressible signal to a low-dimension space, but also can ensure the loss of useful information will not occur in process of dimension reduction. The commonly used measurement matrixes are random Gaussian measurement matrix, random Benoit measurement matrix, partial Hadamard measurement matrix, partial orthogonal measurement matrix, sparse random measurement matrix, Toeplitz and loop measurement matrix, etc. this paper is going to apply random Gaussian measurement matrix, its design approach is: to construct a matrix $\Phi$ size $M \times N$ make every element of $\Phi$ independently subject to a Gaussian distribution which mean is zero, variance is $1 / M$ :

$\Phi_{\mathrm{i}, j} \sim N(0,1 / M)$

This measurement matrix has strong randomness, in the process of compression sensing, random Gaussian measurement matrix is used widely, mainly because random Gaussian matrix is irrelevant to the most of orthogonal basis and orthogonal dictionary, and the number of measurements needed for precise reconstructing is small.

\subsection{Reconstruction of Satellite Cloud Image}

After above sparse processing, the following important part is to reconstructing the satellite cloud picture. MP algorithm is a kind of greedy iterative algorithms, and also is a commonly used algorithm for signal sparse reconstruction. OMP algorithm is a improved algorithm compare with MP algorithm, it has its special significance for all kinds of later greedy algorithms. OMP still use domain selection criteria from MP algorithm, the difference is OMP algorithm use Gram-Schmidt orthogonalization method to orthogonal process selected atoms, then project signals on space which formed by these orthogonal atoms, in order to gain component and iteration surplus of signal in each selected atom, then decompose surplus in the same way. In each step of decomposition, surplus reduced rapidly with decomposition process. Because the selected set of atoms is orthogonalized by recursion, the optimality of iteration is ensured, thus, the numbers of iteration is reduced [6].
On the other hand, because of the orthogonal OMP algorithm [7] will not pick atoms repeatedly, so after finite times of iteration, it could be forced to stop when the times of iteration is achieve sparse degree $\mathrm{K}$. The reconstruction algorithm of OMP is carried out under the condition of limited times of iteration, because of the iterative process is forced to stop, lead more linear measurements are needed to ensure accurate reconstruction. In short, it selects $\Phi$ 's column through greedy iteration method, makes the selected columns from each iteration are associated with current redundant vector to the greatest extent, then minus the relevant part from the measurement vector and iterate repeatedly, until the numbers of iteration achieve sparse degree K.

However, due to the selected atoms need to be GramSchmidit orthogonalization processed, so there is only one atoms will be selected to update the atoms collection in each iteration. Thus, the amount of calculation of the OMP algorithm will be increased greatly, and make its time of signal reconstruction is longer than that of MP algorithm, so, later on, many improved matching pursuit algorithms are emerged, such as ROMP, StOMP, CoSaMP, etc., in order to improve reconstruction efficiency, as well as ensure reconstruction quality.

The original image is picture SCP-01 size $512 \times 512$, through the above steps, the reconstruction images are gain under different sampling rate by experiments, as show in Fig. (4).

Fig. (4) the reconstruction picture of satellite cloud under CS theory combined with wavelet transform and classic OMP algorithm

As it can be seen from Figs. $(\mathbf{1}, \mathbf{5}$ and $\mathbf{6})$ that the quality of reconstruction image is poor and PSNR value is also relatively low when the sampling rate is low, but with the increase of sampling rate, the number of iterations increase, the mean square error (MSE) is gradually reduced.

However, because of the traditional CS theory, wavelet transform and classic OMP algorithm aim at the whole image to carry out sampling calculation and recovery, so that large storage capacity is required, especially for large scale image like satellite cloud image. Furthermore, along with the increase of sampling rate, the numbers of iteration goes up, as well as time-consuming.

From another perspective, this kind of method restoring images after wavelet transform, and structure data through iterative loop, finally synthesize reconstructed images. This method cut apart the correlations between original satellite cloud image matrixes; affect the effect of reconstructed satellite cloud images to a certain extent.

\subsection{The Improvement of Reconstruction Process Algo- rithm for Satellite Image}

Image block compression sensing model can be considered to deal with the problems occurred in the above method, block compressed sensing (BCS) [7] method was proposed by Lu Gan. This method is to divide image into many small blocks, observation and reconstruction is carried out for each 


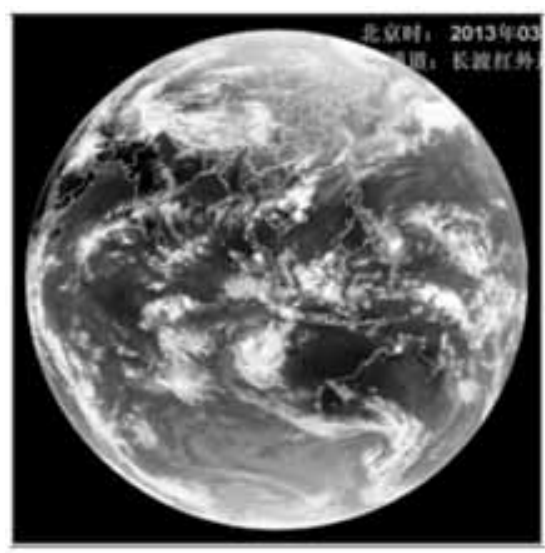

a Original image

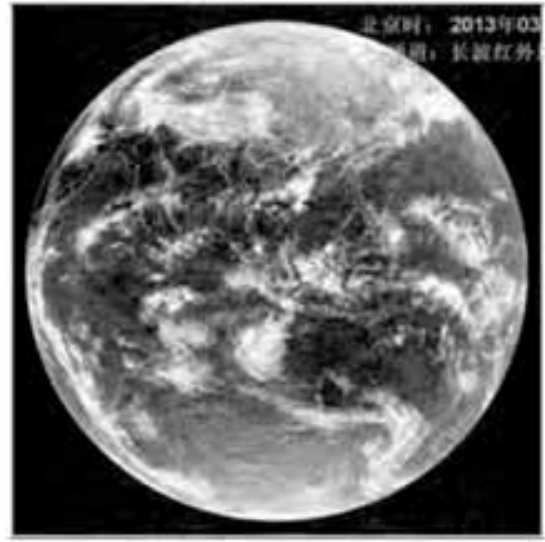

c Sampling rate $\mathrm{M} / \mathrm{N}=0.4$

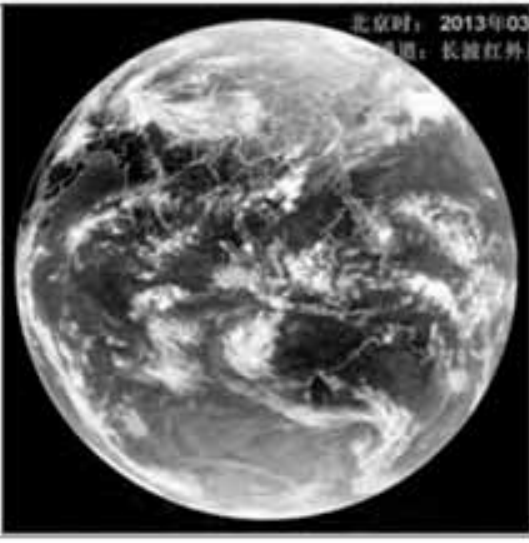

b Sampling rate $\mathrm{M} / \mathrm{N}=0.5$

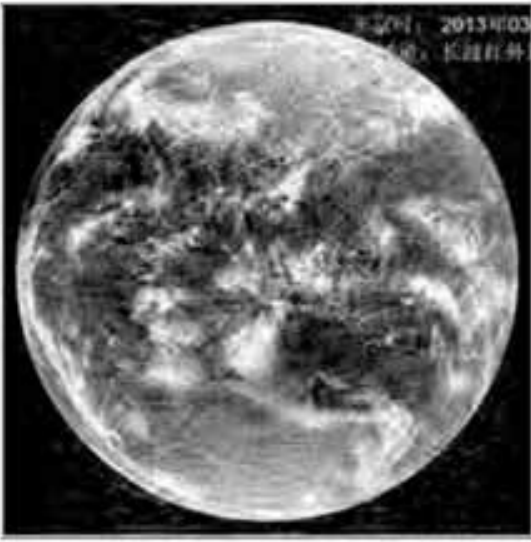

d Sampling rate $\mathrm{M} / \mathrm{N}=0.3$

Fig. (4). The reconstruction picture of satellite cloud under CS theory combined with wavelet transform and classic OMP algorithm.

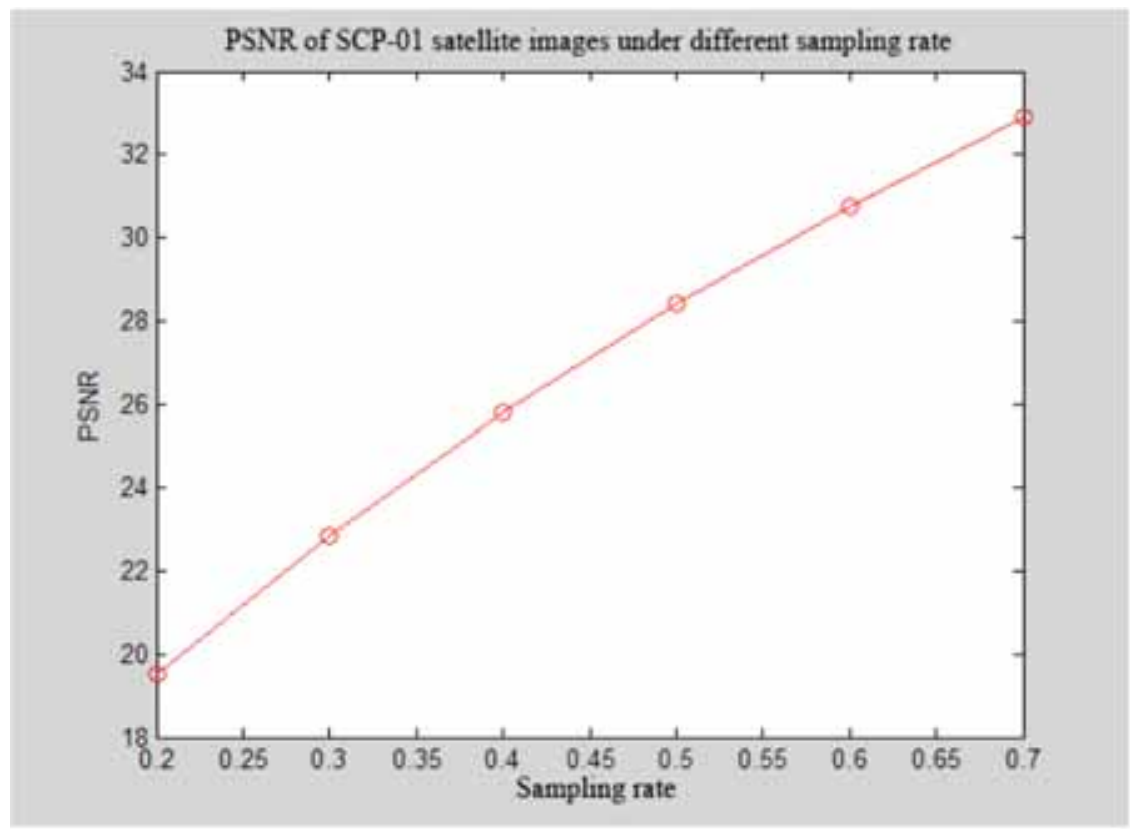

Fig. (5). PSNR of different sampling rate under CS theory combined with wavelet transform and classic OMP algorithm. 


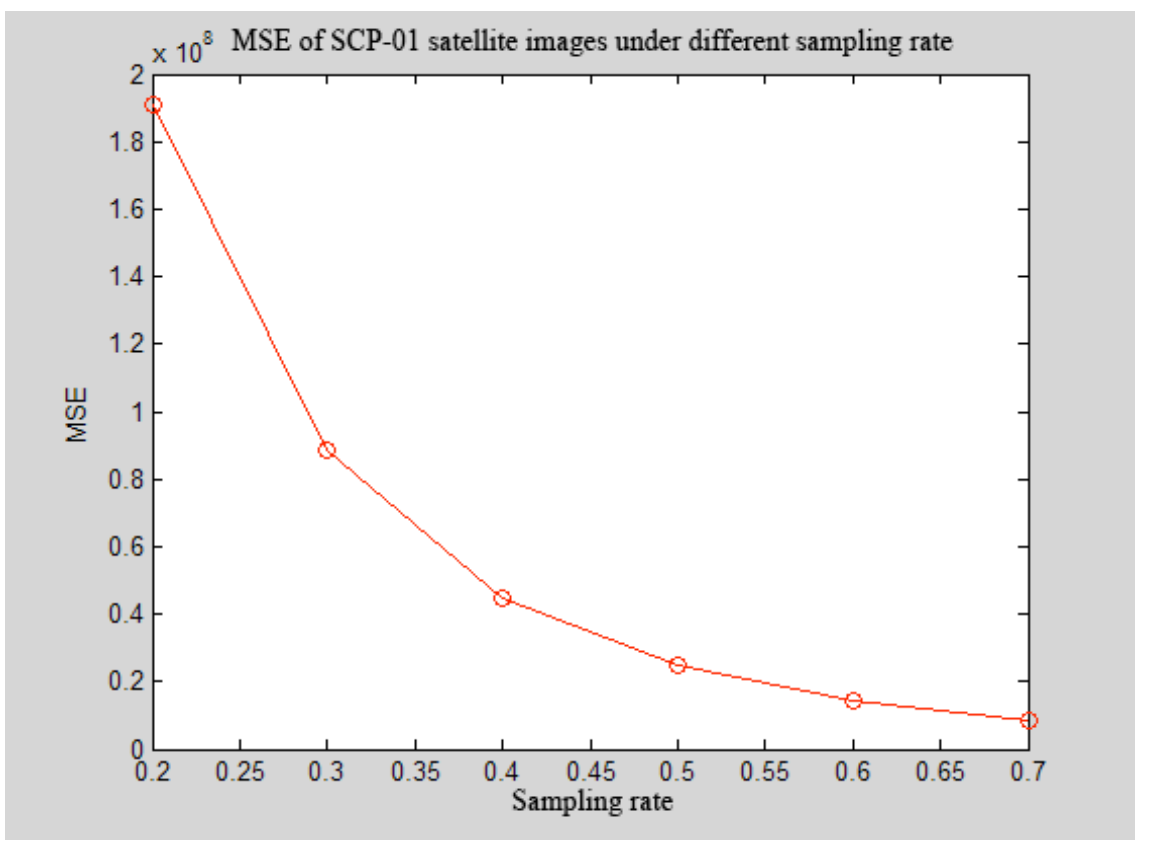

Fig. (6). MSE of different sampling rate under CS theory combined with wavelet transform and classic OMP algorithm.

Table 1. MSE value comparison of reconstructed images under different mean square error.

\begin{tabular}{|c|c|c|c|}
\hline SCP-01(512×512) & Block $(\mathbf{8} \times \mathbf{8})$ Algorithm & Block $(\mathbf{1 6} \times \mathbf{1 6})$ Algorithm & Unblocked OMP Algorithm \\
\hline \hline Mean Square Error (MSE) & & & \\
\hline \hline 0.5 & $2.2037 \mathrm{e}+007$ & $2.3271 \mathrm{e}+007$ & $8.8563 \mathrm{e}+007$ \\
\hline 0.4 & $2.8284 \mathrm{e}+007$ & $2.8251 \mathrm{e}+007$ & $1.9111 \mathrm{e}+008$ \\
\hline 0.3 & $3.5639 \mathrm{e}+007$ & $2.0897 \mathrm{e}+008$ & $5.9532 \mathrm{e}+008$ \\
\hline
\end{tabular}

image block individually, thus, the computational complexity can be greatly reduced. Furthermore, BCS has other advantages, such as small observation matrix which is conductive to storage; once the observed value of each image block is obtained, it can be sent out independently, the receiver can also reconstruct image block independently based on these data, which provides good real-time performance.

Block compressed sensing is descript as follows:

Step 1: partitioning satellite cloud picture SCP-01, pixel value is $X=A \times B$, suppose size of block is $n_{b} \times n_{b}$.

Step 2: apply unified observation matrix $\Phi_{\mathrm{B}}$ to observe each block of satellite cloud image, obtain $\mathrm{M}_{B}$ observation values.

Step 3: scan each block one by one, generate $\mathrm{N}_{B}=\mathrm{n}_{b} \times \mathrm{n}_{b}$ element vectors. Mark the $\mathrm{i}$ block as $\mathrm{Si}=[$ $\left.\mathrm{S}_{\mathrm{i} 1}, \mathrm{~S}_{\mathrm{i} 2}, \ldots, \mathrm{S}_{\mathrm{i} N B}\right]^{\mathrm{T}}$, the observation value of $\mathrm{i}$ block is : $\boldsymbol{Y}_{i}=\Phi_{\mathrm{B}} \mathbf{B}_{S i}$, size of observation matrix $\Phi_{\mathrm{B}}$ is $\mathrm{M}_{B} \times \mathrm{N}_{B}, Y_{i}$ stands for column vector and length is $\mathrm{MB}$, when $\mathrm{M}_{B} \leq \mathrm{N}_{B}$, $\mathrm{M}=\mathrm{M}_{B}(\mathrm{~A} \times \mathrm{B}) / \mathrm{N}_{B}$ observation values can be obtained by observe each block.

Step 4: when observation value of a block is gained, restorative reconstruction could be carried out for this picture block by apply restricting algorithm. The same to every picture blocks, finally, combine each picture blocks and then the whole image is formed. There are many kinds of reconstruction algorithm, and orthogonal matching pursuit is applied in this paper.

The satellite cloud picture SCP-01 which is employed in the paper is size $512 \times 512$, divided as $8 \times 8$ and $16 \times 16$, massive experiments proofs that when the block is smaller than 8 , error of measured signal is relatively big, on the other hand, when block is bigger, calculation is relatively more complex.

\section{THE RESULTS AND COMPARISON OF SIMULA- TIONS}

Satellite cloud picture SCP-01 is used in the experiment, size is $512 \times 512$, and two groups of reconstructed pictures are obtained through BSC process, as shown below:

By analyzing the experimental data, the comparison of MSE, PSNR value of reconstructed satellite cloud picture under different sampling rate by different method are shown in Table $\mathbf{1}$ and Table $\mathbf{2}$ as follows: 
Table 2. PSNR value comparison of reconstructed images under different sampling rate.

\begin{tabular}{|c|c|c|c|}
\hline SCP-01 $(\mathbf{5 1 2} \times \mathbf{5 1 2})$ & block $(\mathbf{8} \times \mathbf{8})$ Algorithm & Block $(\mathbf{1 6} \times \mathbf{1 6})$ Algorithm & Unblocked OMP Algorithm \\
\hline Sampling Rate(M/N) & PSNR/dB & PSNR/dB & PSNR/dB \\
\hline \hline 0.5 & 30.0009 & 32.7487 & 28.3888 \\
\hline 0.4 & 28.8058 & 30.8847 & 25.7879 \\
\hline 0.3 & 27.2307 & 28.7970 & 22.8437 \\
\hline
\end{tabular}

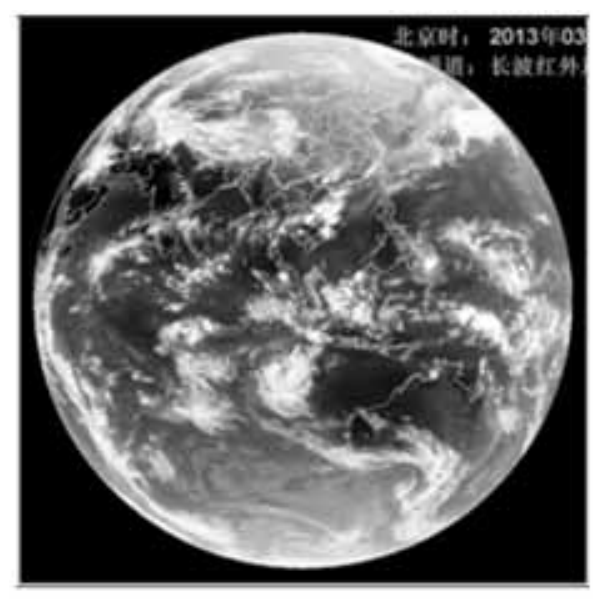

a Original image

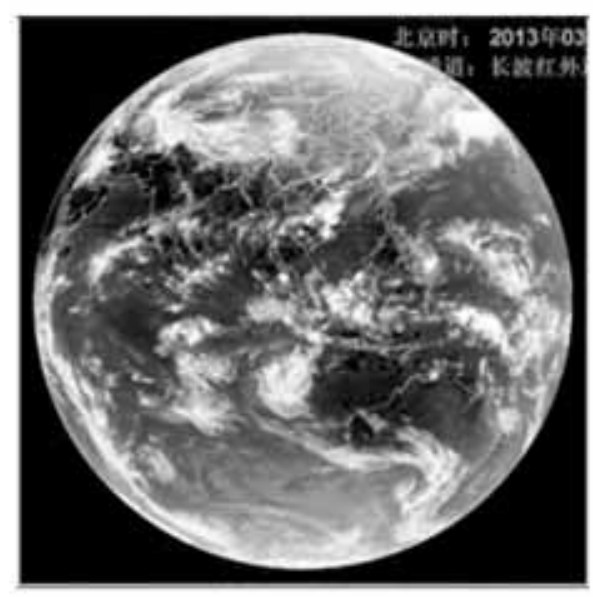

c Sampling rate $\mathrm{M} / \mathrm{N}=0.4$

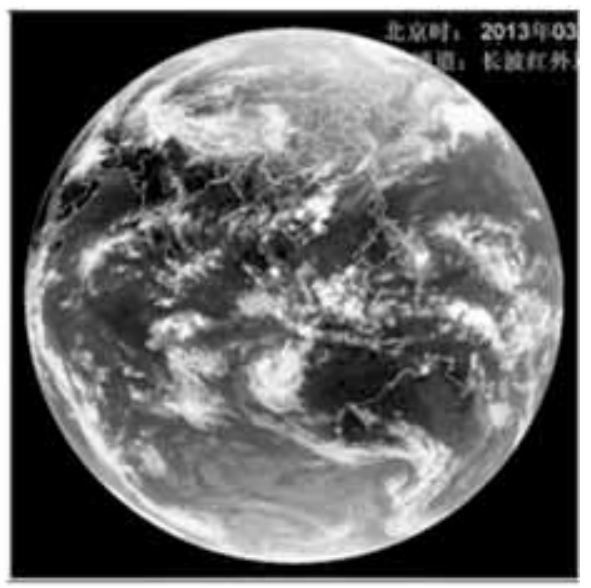

b Sampling rate $\mathrm{M} / \mathrm{N}=0.5$

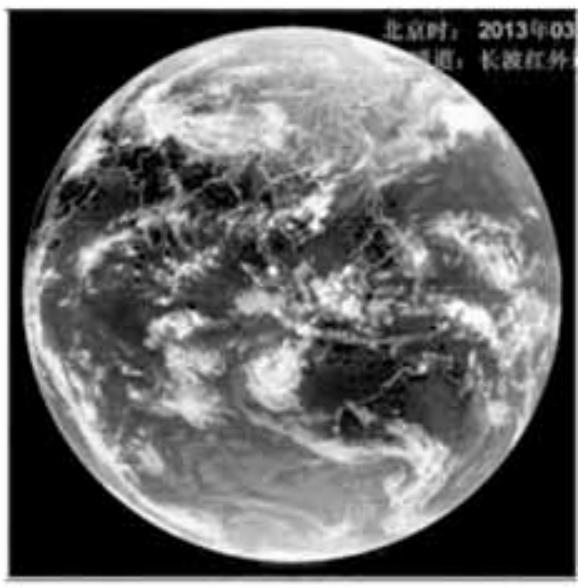

d Sampling rate $\mathrm{M} / \mathrm{N}=0.3$

Fig. (7). Reconstructed images of block size $8 \times 8$ under different sampling rate.

It is can be seen through reconstructed satellite cloud picture that along with the increase of sampling rate, the effect of picture reconstruction is reinforced, as well as the PSNR value of reconstructed image is increased. Figs. (1, 4, 7, and 8) show that quality of image reconstruction has improved obviously after block processing. It is also can be seen from Table 1 that compare with traditional OMP algorithm, the improved algorithm which applied in this paper has outstanding improvement on signal to noise ratio, and with the increase of block size, the PSNR value is relatively increased, the reconstruction effect is improved obviously, but in a large number of experiments, along with the increase of block size, calculation time and complexity is also increased correspondingly. Furthermore, when the sampling rate is too low, obvious blocking effects and man-made noise would emerge, make poor quality image. 


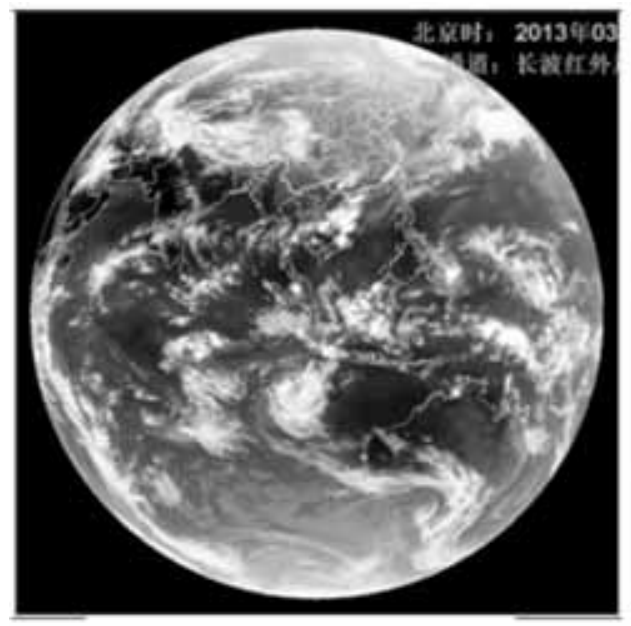

a Original image

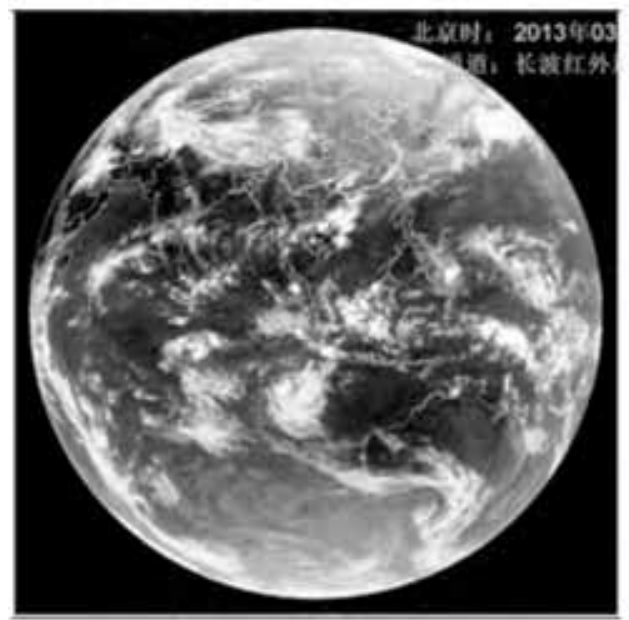

c Sampling rate $\mathrm{M} / \mathrm{N}=0.4$

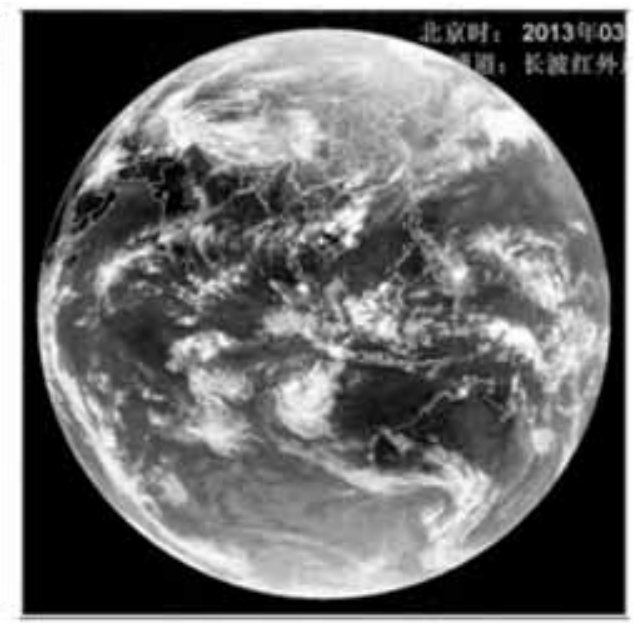

b Sampling rate $\mathrm{M} / \mathrm{N}=0.5$

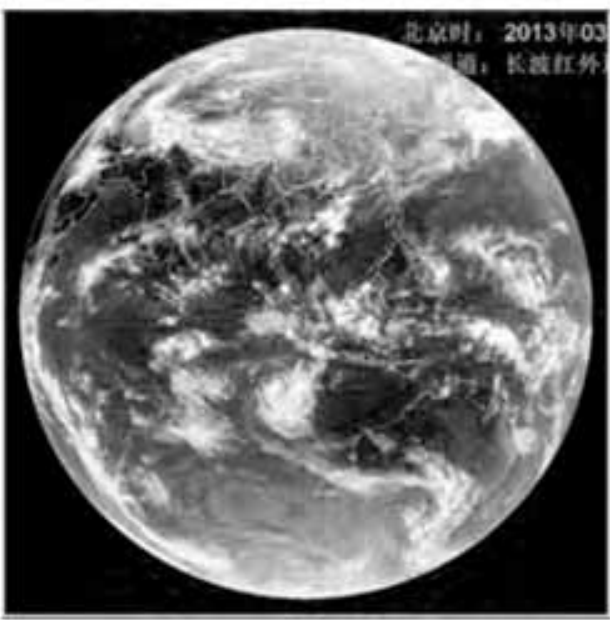

Sampling rate $\mathrm{M} / \mathrm{N}=0.3$

Fig. (8). Reconstructed images of block size $8 \times 8$ under different sampling rate.

\section{CONCLUSION}

This paper has studied the traditional compression sensing theory in the beginning, then carried out satellite cloud image reconstruction by apply greedy iterative algorithmorthogonal matching pursuit algorithm (OMP) of compression sensing theory; and finally, Block Compressed Sensing (BCS) algorithm that apply to satellite cloud image has been proposed. This algorithm not only improves the quality of reconstructed image, but also, the reconstruction effect is superior to the traditional compression sensing algorithm, at the same time, less number of observe value is required, and the objective visual effect of image is markedly superior to the traditional ones. From the perspective of PSNR and MSE value of reconstructed image, the improved approach that proposed in this paper also provided a great progress in reconstruction effect. However, due to the improved algorithm employed the same observation matrix $\Phi_{B}$ on blocking process of satellite cloud picture, there was lack of different weighting for measurement matrix elements value in different frequency of one picture block, consequently lowered the accuracy of low-frequency content-- the main frequency content which influences the image quality, leaded visual effect decrease to a certain degree. In addition, the emergence of blocking effect and man-made noise under the situation of low sampling rate can be counted as insufficiency of this algorithm that can be improved in the future.

\section{CONFLICT OF INTEREST}

The authors confirm that this article content has no conflict of interest.

\section{ACKNOWLEDGEMENTS}

Declared none. 


\section{REFERENCES}

[1] D. Donoho, "Compressed sensing", IEEE Trans. Inform. Theory, vol. 52, no. 4, pp. 1289-1306, 2006.

[2] E. Candès, "Compressive sampling", In: Proc. Int. Cong. Mathe., Madrid, Spain: European Mathematical Society, 2006, pp. 14331452.

[3] S. Mallat, "Multifrequency channel decomposition of images and wavlet models", IEEE Trans. Acoust. Speech Signal Process, vol. 37, no. 12, pp. 2091-2110, 1989.

[4] D. Taubman, and A. Zakhor, "Mulirate 3-D subband coding of video", IEEE Trans. Image Process, vol. 3, no. 5, pp. 572-588, 1994.
[5] Z. Xiong, K. Ranchandran, M.T. Orchard, and Z. Ya-Qin, "A comparative study of DCT-and wavelet-based images coding", IEEE Trans. Circuits Syst. Video Technol., vol. 9, no. 5, pp. 692-695, 1999.

[6] E. Candès, and J. Romberg, "Sparsity and incoherence in compressive sampling”, Inverse Probl., vol. 23, no. 3, pp. 969- 985, 2007.

[7] J. A. Tropp, and A. C. Gilbert, "Signal recovery from Rando-M measurements via orthogonal matching pursuit", IEEE Trans. Inf. Theory, vol. 53, no. 12, pp. 4655- 4666, 2007.

Received: December 15, 2014

(C) Feng et al.; Licensee Bentham Open.

This is an open access article licensed under the terms of the Creative Commons Attribution Non-Commercial License (http://creativecommons.org/licenses/by-nc/3.0/) which permits unrestricted, non-commercial use, distribution and reproduction in any medium, provided the work is properly cited. 